\title{
Prevalencia de Hipertensión Arterial en una unidad de cuidados intensivos neonatales
}

\section{Prevalence of Arterial Hypertension in a neonatal intensive care unit}

\author{
Alejandro Balestracci ${ }^{a}$, Marina Andrea Capone ${ }^{a}$, Ismael Toledo ${ }^{a}$, Sebastián Sticotti ${ }^{b}$ \\ aUnidad de Nefrología. Hospital General de Niños Pedro de Elizalde. Ciudad Autónoma de Buenos Aires. Argentina \\ bUnidad de Neonatología. Hospital General de Niños Pedro de Elizalde. Ciudad Autónoma de Buenos Aires. Argentina
}

Recibido: 22 de junio de 2020; Aceptado: 19 de agosto de 2020

¿Qué se sabe del tema que trata este estudio?

En estudios realizados en Estados Unidos y Europa la prevalencia de hipertensión arterial neonatal (HTN) en las unidades de cuidados intensivos neonatales (UCIN) varía entre el 3 y el 9\%; y se produce predominantemente en pacientes gravemente enfermos.
¿Qué aporta este estudio a lo ya conocido?

En este estudio la prevalencia de HTN en la UCIN fue de 4,7\% y en todos los casos se presentó en pacientes prematuros con factores previamente reconocidos como asociados a esta condición. De esta forma, se agrega información actualizada sobre HTN proveniente de Latinoamérica.

\section{Resumen}

La prevalencia de hipertensión arterial neonatal en las unidades de cuidados intensivos neonatales (UCIN) varía entre el 3 y el 9\%, sin embargo, no existe información actualizada de Latinoamérica. Objetivo: Estimar la prevalencia de hipertensión arterial y evaluar su asociación con causas previamente relacionadas con esta condición. Pacientes y Método: Estudio transversal que incluyó a todos los niños internados en una UCIN durante un año, excluidos aquellos trasladados a cirugía cardiovascular. Se registraron variables maternas y neonatales, hipertensión arterial materna, vía de parto, edad gestacional, edad, sexo, peso de nacimiento, Apgar, antecedente de maduración pulmonar con corticoides y cateterismo de vasos umbilicales. Se consignó motivo de ingreso a UCIN, medicamentos y complicaciones durante la hospitalización. La tensión arterial se registró con oscilómetro automatizado considerando hipertensión arterial según tablas para edad gestacional. La prevalencia se expresó como porcentaje (intervalo de confianza 95\%, IC95\%). La estadística descriptiva se presenta como mediana (rango) o frecuencia de presentación (porcentajes) y se buscó asociación con el Test de Wilcoxon, $\mathrm{Chi}^{2}$ o Fisher según correspondiera $(\mathrm{p}<0,05)$. Resultados: Se reclutaron 169 pacientes (60\% sexo masculino). Edad gestacional: 38 semanas (rango 26-42), 38\% prematuros. Peso $3.000 \mathrm{~g}$ (rango 545-4.950), 32\% bajo peso. Ocho pacientes presentaron hipertensión arterial (prevalencia

Palabras clave: Hipertensión Arterial; Unidad de Cuidados Intensivos Neonatales; Neonatos; Prematuros

Correspondencia:

Alejandro Balestracci

abalestracci@yahoo.com.ar 
4,7\%, IC95\% 2,4-9). La presencia de hipertensión arterial se asoció con prematurez ( $p=0,0003)$, bajo peso $(\mathrm{p}=0,01)$, maduración pulmonar con corticoides $(\mathrm{p}=0,002)$, cateterismo umbilical $(\mathrm{p}=0,03)$, uso de $\geq 2$ drogas nefrotóxicas $(p=0,02)$, tratamiento con cafeína $(\mathrm{p}=0,0001)$, injuria renal aguda $(\mathrm{p}=0,02)$ e hipertensión intracraneal $(\mathrm{p}=0,04)$. Solo un paciente requirió medicación antihipertensiva y en todos los casos se normalizó durante el seguimiento. Conclusión: La prevalencia de hipertensión arterial neonatal fue de 4,7\% y en todos los casos se presentó en niños prematuros con factores previamente reconocidos como asociados a esta condición.

\section{Abstract}

The prevalence of neonatal hypertension in neonatal intensive care units (NICU) ranges between 3 and $9 \%$. However, there is no current data on Latin America. Objective: To estimate the prevalence of neonatal hypertension and to assess its association with causes previously related to this condition. Patients and Method: cross-sectional study. All patients admitted to the NICU during one year were included, excluding those transferred to the cardiovascular NICU. The following maternal and neonatal variables were registered: maternal arterial hypertension, type of delivery, gestational age, age, sex, birth weight, Apgar score, history of pulmonary maturation with corticosteroids, and umbilical vessel catheterization as well as the reason for admission to the NICU, medications, and complications during hospitalization. Blood pressure was measured with an automated oscillometric device, defining neonatal hypertension according to standards in gestational age. Prevalence was expressed as percentage (confidence interval 95\%, CI95\%). Descriptive data were reported as median (range) and frequency of presentation (percentage). Finally, we used the Wilcoxon, $\mathrm{Chi}^{2}$ o Fisher exact test to identify factors related to $\mathrm{NH}$ as applicable $(\mathrm{p}<0.05)$. Results: 169 patients were included (60\% males). Gestational age was 38 weeks (range 26-42 weeks), 38\% were preterm. Birth weight was $3000 \mathrm{~g}$ (range 545-4950 g) and 32\% presented low birth weight. Eight patients presented hypertension during hospitalization (4.7\% prevalence, CI95\% 2.4-9). The presence of hypertension was associated with prematurity $(\mathrm{p}=0.0003)$, low birth weight $(\mathrm{p}=0.01)$, prenatal corticosteroid treatment $(p=0.002)$, umbilical catheterization $(p=0.03)$, administration of $\geq 2$ nephrotoxic drugs $(\mathrm{p}=0.02)$, caffeine treatment $(\mathrm{p}=0.0001)$, acute kidney injury $(\mathrm{p}=0.02)$, and intracranial hypertension $(\mathrm{p}=0.04)$. Only one patient required antihypertensive pharmacologic treatment and in all cases, hypertension was resolved during follow-up. Conclusion: Prevalence of neonatal hypertension in our NICU was $4.7 \%$ and in all cases occurred in preterm newborns with previously recognized factors associated with this condition.

\section{Keywords:}

Arterial Hypertension; Newborn; Neonatal Intensive Care Units; Preterm Newborns

\section{Introducción}

La hipertensión arterial en niños internados en las unidades de cuidados intensivos neonatales (UCIN) es una entidad que puede aumentar su morbimortali$\mathrm{dad}^{1,2}$. La prevalencia de hipertensión arterial neonatal (HTN) en UCIN es del 3\%, pero ante ciertas condiciones, como displasia broncopulmonar, cardiopatías, hemorragia intraventricular y cateterismo de los vasos umbilicales puede alcanzar el 9\% $\%^{3-6}$. Otras situaciones que también se asocian a mayor prevalencia de HTN son el uso de corticoides prenatales, la prematurez, la injuria renal aguda (IRA) y la patología renal intrínseca $^{1,7,8}$. Esta información proviene de estudios realizados en Estados Unidos y en Europa, sin embargo, existe muy escasa información de Latinoamérica sobre su prevalencia; según nuestro conocimiento, sólo un estudio realizado en Chile en el año 1987 comunicó una prevalencia de 2,5\%9. Debido a los grandes avances tecnológicos aplicados al cuidado de los neonatos enfermos, entre los que se incluyen los dispositivos utilizados para el registro de la tensión arterial (TA), sumado a la disponibilidad de tablas con valores de referencia de TA para este grupo etario ${ }^{1}$, conocer la magnitud actual de esta condición podría ser de utilidad en la atención de estos pacientes. El objetivo primario del presente estudio fue estimar la prevalencia de HTN en la UCIN de nuestro hospital. En forma secundaria, nos propusimos explorar la asociación de HTN con causas previamente relacionadas con esta condición y, finalmente, describir el tratamiento y la evolución hasta el egreso de la UCIN de los pacientes hipertensos.

\section{Pacientes y Método}

Estudio transversal en el que se incluyeron todos los pacientes que ingresaron a la UCIN del Hospital General de Niños Pedro de Elizalde en el período comprendido entre el 1 de junio de 2017 y el 31 de mayo 
de 2018. Esta institución recibe niños con patología compleja derivados de otros centros, como también pacientes sin antecedentes perinatológicos que se internan por diversas intercurrencias, principalmente infecciosas. Se excluyeron los niños con cardiopatías congénitas que requirieron ser trasladados a la unidad de terapia cardiovascular para tratamiento quirúrgico.

Se registraron los siguientes datos de las historias clínicas y por interrogatorio materno: antecedente de hipertensión arterial materna, vía de parto, edad gestacional, edad, sexo, peso de nacimiento, puntuación de Apgar, antecedente de maduración pulmonar con corticoides y cateterismo de vasos umbilicales. Se consignó también el motivo de ingreso a UCIN y complicaciones durante la internación como infecciones intrahospitalarias, IRA, hemorragia intraventricular e hipertensión intracraneal (HTI). Además, se registraron las drogas que recibieron, especialmente nefrotóxicos (antibióticos, indometacina, etc.) y otras asociadas a HTN (inotrópicos, cafeína) ${ }^{7,10}$.

El registro de la TA fue realizado diariamente en todos los pacientes por un integrante del equipo de investigación según el protocolo propuesto por Nwankwo y colaboradores ${ }^{11}$. Siguiendo el mismo, se ubicó al recién nacido en posición prona o supina, respetando un intervalo de $1,5 \mathrm{~h}$ después de alimentación o intervención médica, con colocación del manguito apropiado en el brazo superior derecho. Posteriormente, se mantuvo al niño sin estímulo por 15 min y luego, estando dormido o en vigilia tranquila, se realizaron 3 lecturas de TA sucesivas a intervalos de $2 \mathrm{~min}$. Dado que el primer registro tiende a ser habitualmente más alto, consideramos para este estudio el valor promedio de las tres mediciones ${ }^{11}$. Si bien se han utilizado otras formas para medir los valores de TA en este grupo etario ${ }^{12}$, en este estudio los registros se realizaron con dispositivo oscilométrico ya que sus determinaciones guardan buena correlación con la medición de la TA intraarterial y a que las tablas de valores normales fueron construidas en base a esta metodología ${ }^{1,7,13}$. En el caso de los pacientes en estado crítico se utilizó Monitor Philips Intelli Vue MP40 ${ }^{\circledR}$ y en aquellos de menor complejidad Monitor Philips Sure Signs VSi ${ }^{\circledR}$, esto se debió a que la UCIN se encuentra dividida físicamente en 2 sectores (alta y moderada complejidad) y que por cuestiones de asepsia los aparatos no se intercambian entre dichas áreas. Ambos son modelos con método oscilométrico de desinsuflación escalonada, comparten los manguitos y pertenecen al mismo fabricante. Los monitores fueron calibrados en forma periódica por técnicos de la empresa proveedora. En base a los registros obtenidos se consideraron hipertensos a aquellos pacientes que mantuvieran registros elevados por $24 \mathrm{~h}$ o más para evitar clasificar como tales los que hubieran tenido aumentos transitorios sin significado clínico (por ejemplo relacionados con llanto, alimentación, etc. $)^{1,11}$. Cabe mencionar que además de las tomas de TA pautadas por el protocolo de estudio, el personal de enfermería realizó las determinaciones de TA cada 4-6 h correspondientes a la evaluación de rutina de signos vitales de los pacientes en UCIN. A pesar de que en este contexto generalmente se realiza un único registro, a diferencia de las 3 mediciones recomendadas ${ }^{11}$; durante el período de estudio no se intentó influir o modificar la técnica utilizada habitualmente. Además, debido a que ha sido observado que los registros rutinarios de TA realizados por el personal de enfermería suelen ser significativamente mayores que los obtenidos en forma protocolizada ${ }^{11}$, aquellos pacientes que presentaron registros de hipertensión arterial en los controles de rutina fueron evaluados repetidamente por el equipo de investigación para asegurar una adecuada clasificación del paciente.

\section{Definiciones operativas}

- HTN: valores de TA > percentilo 95 para edad y sexo según lo establecido por Dionne y col, sostenidos por más de $24 \mathrm{~h}^{1}$.

- Según la edad gestacional los pacientes fueron clasificados en recién nacidos de término (entre 38 y 40 semanas) y pretérmino ( $\leq 37$ semanas completas). A su vez, se consideraron las siguientes subcategorías de nacimiento prematuro: 1) extremadamente prematuro ( $<28$ semanas), muy prematuro $(28$ a $<32$ semanas) y prematuro moderado o tardío (32 a $\leq 37$ semanas completas) $)^{14,15}$.

- El peso al nacer se relacionó con la EG y en base a las tablas nacionales, se los subdividió en peso elevado, peso adecuado y bajo peso ${ }^{14,16}$.

- IRA: fue definida según criterios KDIGO modificados para neonatos según caída del filtrado glomerular o de diuresis. Consideramos IRA del estadio 1 en adelante ${ }^{17,18}$.

\section{Consideraciones éticas}

El estudio fue aprobado por los Comités de Investigación y de Ética de nuestra institución. Se obtuvo consentimiento informado de los padres y/o tutores de los pacientes para incluirlos en este protocolo de estudio.

\section{Análisis estadístico}

El tamaño muestral se calculó para prevalencia, estimándose en 139 pacientes considerando una prevalencia promedio de HTN de 4,5\% (confianza 95\%, precisión $2 \%)^{1-6}$. Las variables continuas no se ajustaron a normalidad (Test de Shapiro-Wilk) por lo que fueron expresadas como mediana (rango), en tanto que las variables categóricas se informaron según frecuencia de presentación. Se tomó la TA en forma diaria 
a todos los niños internados para establecer la prevalencia de HTN, la cual fue expresada como porcentaje con su respectivo intervalo de confianza de 95\% (IC 95\%).

Para evaluar la asociación entre las condiciones seleccionadas (sexo, prematurez, bajo peso al nacer, puntaje de Apgar, vía de parto, hipertensión arterial materna, maduración pulmonar con corticoides, cateterismo umbilical, patología renal, drogas recibidas, hidrocefalia con HTI y patología quirúrgica) e HTN, los pacientes fueron divididos en dos grupos (con y sin HTN). Dado que las variables cuantitativas carecieron de distribución normal se compararon con el Test de Wilcoxon, en tanto que los datos categóricos se evaluaron con el test de Chi cuadrado o de Fisher, según correspondiera. Un valor de $\mathrm{p}<0,05$ (2 colas) se consideró estadísticamente significativo. Los datos fueron analizados con el programa Statistix 7 (IBM version; Analytical Software, Tallahasee, FL).

\section{Resultados}

Se reclutaron 173 pacientes, 4 de los cuales se excluyeron porque fueron derivados a la unidad de terapia cardiovascular, quedando la muestra de estudio conformada por 169 pacientes (figura 1). El 74\% de los pacientes incluidos había nacido por parto vaginal y 101 $(60 \%)$ correspondieron al sexo masculino. La mediana de la edad fue de 8 días (rango 1-28 días), con una mediana de edad gestacional (EG) de 38 semanas (rango 26-42 semanas), siendo 64 (38\%) prematuros. Dentro de estos últimos, 6 eran prematuros extremos, 15 muy prematuros y 43 prematuros moderados y tardíos. La mediana de peso fue $3.000 \mathrm{~g}$ (rango 545-4.950 g) con

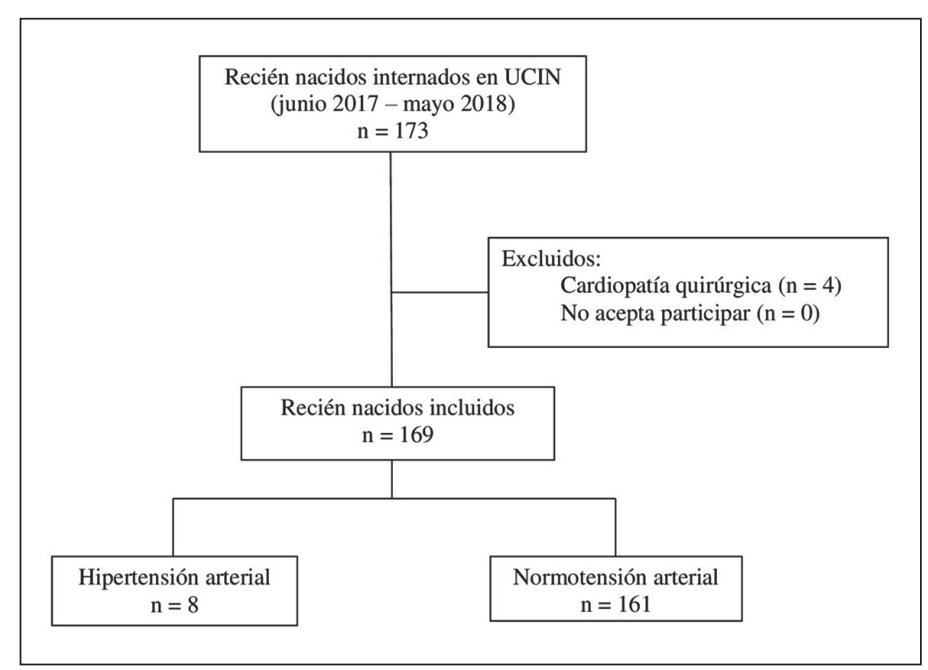

Figura 1. Selección de pacientes en Unidad de Cuidados Intensivos neonatal (UCIN). una distribución del 65,7\% para peso adecuado, 32\% bajo peso y 2,3\% para alto peso.

Los motivos de internación se describen en la tabla 1. Los más frecuentes fueron la patología quirúrgica $(41,4 \%)$, las intercurrencias respiratorias $(19,5 \%)$, las infecciones adquiridas en la comunidad $(14,8 \%)$ y BRUE (de la nomenclatura inglesa Brief Resolved Unexplained Events) en el 10,1\% de los casos. Durante la internación se observaron las siguientes complicaciones: infecciones intrahospitalarias $(33,1 \%)$, desarrollo de IRA $(17 \%)$, de HTI $(4,7 \%)$ y óbito $(4,7 \%)$. La prevalencia de hemorragia intraventricular en prematuros fue de $15,6 \%$, alcanzando el $33 \%$ en pacientes con peso inferior a $1.500 \mathrm{~g}$.

Se detectó HTN en 8 pacientes, lo que representó una prevalencia de 4,7\% (IC 95\%: 2,4-9). Todos los casos correspondieron a pacientes prematuros (2 extremos, 3 muy prematuros y 3 moderados y tardíos) internados en el subsector de la UCIN de alta complejidad (tabla 2).

Se encontró asociación estadísticamente significativa entre el desarrollo de HTN y las siguientes condiciones: prematurez, bajo peso para la edad gestacional, antecedente de maduración pulmonar con corticoides y de cateterismo umbilical, haber recibido $\geq 2$ drogas nefrotóxicas, el desarrollo de IRA, presencia de HTI y la administración de cafeína (tabla 3). Tres de los pacientes hipertensos tenían hemorragia intraventricular y a cuatro se les había colocado un catéter en los vasos arteriales umbilicales. En todos los casos la HTN se detectó en pacientes inicialmente normotensos que desarrollaron la complicación en el transcurso de la internación (tabla 2). Merece mencionarse que de los 6 pacientes con HTN que recibieron cafeína en 4 casos el aumento de la TA ocurrió

\begin{tabular}{lcc}
\multicolumn{3}{l}{$\begin{array}{l}\text { Tabla 1. Motivos de internación en la unidad de } \\
\text { cuidados intensivos neonatales }\end{array}$} \\
\hline Motivo de internación & \multicolumn{2}{l}{ Pacientes $(\mathrm{n}=169)$} \\
\cline { 2 - 3 } & 70 & 41,4 \\
\hline Patología quirúrgica & 33 & 19,5 \\
Intercurrencia respiratoria & 25 & 14,8 \\
Infección de la comunidad & 17 & 10,1 \\
BRUE & 9 & 5,2 \\
Cardiopatía & 4 & 2,4 \\
Ictericia prolongada & 2 & 1,2 \\
Trastorno metabólico & 2 & 1,2 \\
Síndrome mieloproliferativo & 7 & 4,2 \\
Otros &
\end{tabular}

BRUE: Brief Resolved Unexplained Events. 
Tabla 2. Características demográficas y clínicas de pacientes con hipertensión arterialneonatal

\begin{tabular}{|c|c|c|c|c|c|c|c|c|c|c|c|}
\hline Caso & Sexo & $\begin{array}{c}\text { Edad } \\
\text { gestacional } \\
\text { (semanas) }\end{array}$ & $\begin{array}{l}\text { Peso de } \\
\text { nacimiento } \\
\text { (gramos) }\end{array}$ & $\begin{array}{l}\text { Motivo de } \\
\text { internación }\end{array}$ & $\begin{array}{c}\text { Maduración } \\
\text { pulmonar }\end{array}$ & $\begin{array}{c}\text { Cateterismo } \\
\text { umblical }\end{array}$ & $\begin{array}{l}\text { Injuria } \\
\text { renal } \\
\text { aguda }\end{array}$ & $\begin{array}{c}\text { Drogas } \\
\text { nefrotóxicas } \\
\text { ( } \geq 2 \text { drogas) }\end{array}$ & Cafeína & $\begin{array}{l}\text { Hipertensión } \\
\text { intracraneal }\end{array}$ & Tratamiento \\
\hline 1 & $\mathrm{~F}$ & 37 & 2.060 & $\begin{array}{l}\text { Mielomenin- } \\
\text { gocele }\end{array}$ & No & No & No & No & No & Sí & VDVP \\
\hline 2 & $\mathrm{~F}$ & 28 & 590 & $\begin{array}{l}\text { Malrotación } \\
\text { intestinal }\end{array}$ & Sí & Sí & Sí & Sí & Sí & No & $\begin{array}{c}\text { Medidas } \\
\text { generales* }\end{array}$ \\
\hline 3 & M & 28 & 855 & Cardiopatía & Sí & Sí & No & Sí & Sí & No & $\begin{array}{c}\text { Medidas } \\
\text { generales* }\end{array}$ \\
\hline 4 & $M$ & 30 & 980 & $\begin{array}{l}\text { Enterocolitis } \\
\text { necrotizante }\end{array}$ & Sí & Sí & Sí & Sí & Sí & No & $\begin{array}{c}\text { Medidas } \\
\text { generales* }\end{array}$ \\
\hline 5 & $\mathrm{~F}$ & 37 & 3.240 & Kernicterus & No & No & No & No & No & No & Enalapril \\
\hline 6 & $M$ & 27 & 730 & Onfalocele & Sí & Sí & Sí & Sí & Sí & No & $\begin{array}{c}\text { Medidas } \\
\text { generales* }\end{array}$ \\
\hline 7 & $M$ & 33 & 1.410 & Hidrocefalia & Sí & No & No & Sí & Sí & Sí & VDVP \\
\hline 8 & $\mathrm{~F}$ & 26 & 710 & Cardiopatía & Sí & No & Sí & Sí & Sí & No & $\begin{array}{c}\text { Medidas } \\
\text { generales* }\end{array}$ \\
\hline
\end{tabular}

F: femenino. M: masculino. VDVP: Válvula de derivación ventrículo peritoneal. *Ajuste o suspensión de drogas, corrección de volemia, analgesia.

Tabla 3. Condiciones asociadas en pacientes con hipertensión arterial neonatal

\begin{tabular}{|c|c|c|c|}
\hline Característica & $\begin{array}{l}\text { Hipertensos } \\
\qquad(n=8)\end{array}$ & $\begin{array}{l}\text { Normotensos } \\
\quad(n=161)\end{array}$ & p-valor \\
\hline Sexo (masculino/femenino) & $4 / 4$ & $97 / 64$ & 0,7 \\
\hline Prematurez & 8 & 56 & 0,0003 \\
\hline Bajo peso para la edad gestacional & 6 & 49 & 0,01 \\
\hline Vía de parto (vaginal/cesárea) & $5 / 3$ & $120 / 41$ & 0.68 \\
\hline Puntaje de Apgar $1 \mathrm{~min}$ & $7(6-9)$ & $9(2-9)$ & 0.16 \\
\hline Puntaje de Apgar 5 min & $9(8-10)$ & $10(5-10)$ & 0.27 \\
\hline Hipertensión arterial materna & - & 17 & 0.6 \\
\hline Maduración pulmonar con corticoides & 6 & $17^{\#}$ & 0,002 \\
\hline Cateterismo umbilical & 4 & $22 *$ & 0,03 \\
\hline Hipertensión intracraneal & 2 & 6 & 0,04 \\
\hline Injuria renal aguda & 4 & 25 & 0,02 \\
\hline Uropatía & 3 & 23 & 0,1 \\
\hline$\geq 2$ drogas nefrotóxicas & 6 & 56 & 0,02 \\
\hline Cafeína & 6 & 6 & 0,0001 \\
\hline Inotrópicos & 1 & 21 & 1 \\
\hline Patología quirúrgica & 5 & 65 & 0,27 \\
\hline
\end{tabular}

*Dato disponible en 143 pacientes. "Dato disponible en 142 pacientes.

durante la dosis de ataque y en 2 durante el mantenimiento. En relación al tratamiento, sólo un paciente con edema cerebral secundario a kernicterus requirió enalapril durante 3 semanas por asociación de HTN con síntomas neurológicos; por el contrario, todos los restantes presentaron descenso de los niveles tensionales con medidas de sostén resolviendo el cuadro dentro de las $72 \mathrm{~h}$. 


\section{Discusión}

La prevalencia de HTN en la UCIN de nuestro hospital fue de 4,7\%. Este hallazgo es consistente con estudios previos que observaron una prevalencia entre el 3\% y el $9 \%$ en pacientes críticamente enfermos con múltiples intercurrencias asociadas ${ }^{1-6}$. En nuestra serie todos los pacientes que desarrollaron HTN compartían dichas características clínicas, sin embargo el diseño de la presente investigación permite evaluar asociación pero no causalidad. De todas formas, al tratarse de niños críticamente enfermos, podría especularse que la HTN sea de causa multifactorial. En acuerdo con esto, Dionne y Flynn observaron recientemente que el 75\% de los pacientes con HTN en las UCINs correspondía a niños prematuros con las complicaciones propias de este grupo etario ${ }^{19}$.

En nuestros pacientes, tanto la prematurez como el bajo peso se asociaron significativamente con el desarrollo de HTN. El riñón termina de desarrollarse a finales del tercer trimestre del embarazo, por lo que está especialmente expuesto a alterar su desarrollo normal en caso de nacer en forma prematura. Esta situación puede condicionar, entre otras consecuencias, una menor masa renal funcional y cambios microvasculares que representan un riesgo elevado de hipertensión arterial y daño renal crónico en el largo plazo ${ }^{20}$, como ya fuera demostrado en estudios epidemiológicos ${ }^{21,22}$. Además, la menor masa nefronal determina mayor susceptibilidad al desarrollo de IRA ante eventos de hipoxia/isquemia y de exposición a drogas nefrotóxi$\mathrm{cas}^{23}$. Por consiguiente, a pesar de haber resuelto los eventos asociados a HTN, este grupo de niños requiere seguimiento a largo plazo ${ }^{20,24,25}$.

El cateterismo de vasos umbilicales, especialmente el de arterias umbilicales, es uno de los mayores factores de riesgo para HTN; Watkinson destaca que dicho procedimiento podría relacionarse con el $80 \%$ de los casos de HTN hasta las 2 primeras semanas de vida ${ }^{3}$. A pesar de que la tasa de formación de trombos intravasculares no supera el $25 \%$, otros mecanismos como el estímulo endotelial con vasoconstricción asociada podrían contribuir al desarrollo de $\mathrm{HTN}^{19,24}$. Cuatro de los 8 pacientes hipertensos de nuestra serie tenían como antecedente cateterismo de arterias umbilicales; si bien en ellos no se detectó trombosis de vasos renales por ecografía doppler, es sabido que este es un hallazgo inconstante ${ }^{1,19}$.

Mohammed et al observaron que el 2,5\% de los pacientes que recibían cafeína desarrollaban HTN, hallazgo que no se relacionaba con la dosis utilizada ${ }^{26}$. En nuestra serie 6 de los 12 pacientes que recibieron cafeína para el tratamiento de apneas desarrollaron HTN; en 4 de ellos se produjo mientras recibían la dosis de ataque y en los 2 restantes bajo dosis de mantenimien- to. Ninguno de estos niños alcanzó el percentilo 99 de TA y todos se mantuvieron asintomáticos. Los registros se normalizaron cuando se disminuyó la dosis de droga o cuando se suspendió.

La prevalencia de IRA en nuestra serie fue de $17 \%$, hallazgo observado en todos los casos en pacientes prematuros críticamente enfermos. De manera remarcable, la mitad de los pacientes de nuestra serie con HTN presentaron IRA durante en el curso de la internación. Como ya fuera mencionado, estos pacientes son especialmente susceptibles al daño renal por su baja masa nefronal, sumado a la gravedad de su estado clínico y a la superposición de factores de riesgo durante internaciones que suelen ser prolongadas ${ }^{19}$. En acuerdo con esto, Shalaby y colaboradores demostraron recientemente que los principales factores de riesgo asociados a esta condición son la EG, el bajo peso al nacer, la exposición a nefrotóxicos y la sepsis ${ }^{27}$. Ciertas malformaciones estructurales nefrourológicas como la poliquistosis renal o las uropatías severas suelen cursar con HTN; ${ }^{1}$ si bien en nuestra casuística el 15\% presentó anomalías congénitas nefrourológicas, la mayoría de ellas fueron menores (más frecuentemente dilataciones pielocaliciales) y consecuentemente cursaron con normotensión arterial.

En cuanto al tratamiento farmacológico, no existe consenso respecto al momento óptimo para su inicio $^{14,22,27}$. En pacientes asintomáticos con TA entre percentilo 95 y 99 se buscará corregir los factores coadyuvantes como la corrección de hipervolemia, ajuste de drogas nefrotóxicas e inotrópicos y manejo del dolor; reservando el tratamiento farmacológico para las situaciones en que la TA alcanza el percentilo $99^{14}$. La elección de la droga en estos casos también es controversial, ya que la mayoría no se encuentran aprobadas para el uso en neonatología, esto podría justificar por qué entre el 18 y el 25\% de los pacientes con HTN no reciben medicación antihipertensiva ${ }^{19}$. En nuestra experiencia, 2 pacientes con hidrocefalia severa e HTI presentaron registros de TA mayores al percentilo 99. Ante la emergencia se realizaron punciones evacuatorias con estabilización de la TA hasta la realización de la derivación ventricular definitiva. En el resto de los casos se adoptaron medidas generales como el ajuste de drogas nefrotóxicas en el caso de que coincidiera con IRA, descenso de la dosis o suspensión de cafeína, diuréticos para disminuir la sobrecarga hidrosalina y analgesia en el caso del niño cursando postoperatorio inmediato de onfalocele. Solo una niña con diagnóstico de kernicterus requirió medicación antihipertensiva, la cual se mantuvo durante 3 semanas. A pesar de que en la mayoría de los casos la HTN corresponde a eventos transitorios, como se observó en nuestros pacientes, existen estudios que sugieren que en el seguimiento a largo plazo la prevalencia se mantiene cercana al $1,3 \%{ }^{28}$. 
Este estudio presenta como fortaleza la recolección prospectiva de datos con el registro continuo y sistematizado de la TA a cargo del equipo de investigación, a diferencia de estudios previos en que la metodología de la toma de la TA fue variable en frecuencia y en metodología del registro ${ }^{3-6}$. Además, aporta datos actuales de nuestra región sobre esta condición que pueden ser de utilidad para enfatizar la pesquisa de esta patología así como para el desarrollo de estrategias de prevención y seguimiento a largo plazo de los pacientes afectados. Por otro lado, una potencial limitación de este estudio es que el $41 \%$ de la muestra correspondió a neonatos derivados para resolución de patología quirúrgica; sin embargo, merece mencionarse que esta condición no se asoció significativamente con el desarrollo de HTN. Además, la prevalencia encontrada es coincidente con comunicaciones previas de niños críticamente enfermos y mucho mayor a la observada en recién nacidos $\operatorname{sanos}(0,2 \%)^{2-6}$.

\section{Conclusiones}

La prevalencia de HTN encontrada en la UCIN de nuestro hospital fue de 4,7\% y afectó exclusivamente a niños prematuros con múltiples factores de riesgo. Si bien en todos los casos se resolvió, nuestros hallazgos refuerzan la importancia del control continuo de la TA en este grupo especial de pacientes.

\section{Responsabilidades Éticas}

Protección de personas y animales: Los autores declaran que los procedimientos seguidos se conformaron a las normas éticas del comité de experimentación humana responsable y de acuerdo con la Asociación Médica Mundial y la Declaración de Helsinki.

Confidencialidad de los datos: Los autores declaran que han seguido los protocolos de su centro de trabajo sobre la publicación de datos de pacientes.

\section{Derecho a la privacidad y consentimiento informa-} do: Los autores han obtenido el consentimiento informado de los pacientes y/o sujetos referidos en el artículo. Este documento obra en poder del autor de correspondencia.

\section{Conflicto de intereses}

Los autores declaran no tener conflicto de intereses.

\section{Financiamiento}

Beca de Investigación de la Sociedad Argentina de Pediatría.

\section{Referencias}

1. Dionne J, Abitbol C, Flynn J. Hypertension in infancy: diagnosis, management and outcome. Pediatr Nephrol. 2012; 27(1):17-32.

2. American Academy of Pediatrics Committee on Fetus and Newborn. Routine evaluation of blood pressure, hematocrit and glucose in newborns. Pediatrics. 1993; 92(3):474-6.

3. Watkinson M. Hypertension in the newborn baby. Arch Dis Child Fetal Neonatal Ed. 2002; 86(2):F78-F88.

4. Buchi KF, Siegler RL. Hypertension in the first month of life. J Hypertens. 1986; 4(5):525-8.

5. Skalina MEL, Kliegman RM, Fanaroff AA. Epidemiology and management of severe symptomatic neonatal hypertension. Am J Perinatol. 1986; 3(3):235-9.

6. Singh HP, Hurley RM, Myers TF. Neonatal hypertension: incidence and risk factors. Am J Hypertens. 1992; 5(2):51-5.

7. Flynn J. Hypertension in the neonatal period. Curr Opin Pediatr. 2012; 24(2):197-204.

8. Seliem WA, Falk MC, Shadbolt B, Kent
AL. Antenatal and postnatal risk factors for neonatal hypertension and infant follow-up. Pediatr Nephrol. 2007; 22(12):2081-7.

9. Norero V C; Concha A; Hubach E, Galdámes, J. Arterial hypertension among admissions to intensive neonatal care. Rev. chil. Pediatr. 1987; 58(1):53-7.

10. Hanna MH, Askenazi DJ, Selewski DT. Drug-induced acute kidney injury in neonates. Curr Opin Pediatr. 2016; 28(2):180-7.

11. Nwankwo M, Lorenz J, Gardiner J. A standard protocol for blood pressure measurement in the newborn. Pediatrics. 1997; 99(6):E10.

12. Lagomarsino F Edda, von Dessauer $G$ Bettina, Molina M Helia, Solar G Eric, Gajardo L Rodrigo. Medición de presión arterial con Doppler en recién nacidos y lactantes normales. Rev. Chil. Pediatr. 1989; 60 (1):10-4.

13. Batisky D. Neonatal Hypertension. Clin Perinatol. 2014; 41(3):529-42.

14. Rodríguez D. Seguimiento de Prematuros. En: Programa Nacional de Actualización Pediátrica Sociedad Argentina de Pediatría. 2013;69-101.
15. Shapiro-Mendoza CK, Lackritz EM. Epidemiology of late and moderate preterm birth. Semin Fetal Neonatal Med. 2012; 17(3):120-5.

16. Comité Nacional de Crecimiento y Desarrollo: Concepto y uso de los estándares de crecimiento. En: Guía para la evaluación del crecimiento físico, Buenos Aires: Editorial Fundasap. Tercera edición. 2013: 27-97.

17. Selewski DT, Charlton JR, Jetton JG, et al. Neonatal Acute Kidney Injury. Pediatrics. 2015; 136(2):e463-e473.

18. Group AKIW. KDIGO Clinical Practice Guideline for Acute Kidney Injury. Kidney Int. 2012;2:S1-S138.

19. Dionne JM, Flynn JT. Management of severe hypertension in the newborn. Arch Dis Child. 2017;102(12):1176-9.

20. Cavagnaro F. The kidney of the premature child: long-term risks. Rev Chil Pediatr. 2020;91(3):324-9.

21. Chehade H, Simeoni U, Guignard JP, Boubred F. Preterm Birth: Long Term Cardiovascular and Renal Consequences. Curr Pediatr Rev. 2018;14(4):219-26.

22. Abitbol CL, Rodriguez MM. The long-term renal and cardiovascular 
consequences of prematurity. Nat Rev Nephrol. 2012;8(5):265-74.

23. Nada A, Bonachea EM, Askenazi DJ. Acute kidney injury in the fetus and neonate. Semin Fetal Neonatal Med. 2017; 22(2):90-7.

24. Nickavar A, Assadi F. Managing hypertension in the newborn infants. Int $\mathrm{J}$ Prev Med. 2014;5(1):S39-43.

25. Starr MC, Flynn JT. Neonatal hypertension: cases, causes, and clinical approach. Pediatr Nephrol. 2019;34(5):787-99.

26. Mohammed S, Nour I, Shabaan AE, Shouman B, Abdel-Hady H, Nasef N. High versus low-dose caffeine for apnea of prematurity: a randomized controlled trial. Eur J Pediatr. 2015;174(7):949-56.

27. Shalaby MA, Sawan ZA, Nawawi
E, Alsaedi S, Al-Wassia H, Kari JA. Incidence, risk factors, and outcome of neonatal acute kidney injury: a prospective cohort study. Pediatr Nephrol. 2018;33(9):1617-24.

28. Taylor H, Kleine I, Bewley S, Loucaides E, Sutcliffe A. Neonatal Outcomes of Waterbirth: A Systematic Review and Meta-Analysis. Arch Dis Child Fetal Neonatal Ed. 2016;101(4):F357-65 